\title{
Studying pharmaceutical tablets mixing process inside a perforated pan- coater using in-line terahertz sensing
}

\author{
Xiaoran $\mathrm{Li}^{1}$, Prince Bawuah ${ }^{2}$, Bryan M. Williams ${ }^{3}$, J. Axel Zeitler ${ }^{2}$, Hungyen Lin ${ }^{1}$ \\ ${ }^{1}$ Department of Engineering, Lancaster University, United Kingdom \\ ${ }^{2}$ Department of Chemical Engineering and Biotechnology, University of Cambridge, United Kingdom \\ ${ }^{3}$ Department of Computing and Communications, Lancaster University, United Kingdom
}

\begin{abstract}
In-line terahertz sensing has been demonstrated to measure the coating thickness of individual pharmaceutical tablets during coating operation. As coating uniformity is highly dependent on tablet mixing, this study presents our research progress to date on using in-line terahertz sensing to investigate the effects of baffle design, drum rotational speed and batch size, on tablet mixing inside a laboratory-sized perforated pan coater.
\end{abstract}

\section{INTRODUCTION}

Pharmaceutical film coating can serve the purpose of prolonging shelf life of the active ingredient or improving the aesthetics of the dosage forms. It can also serve a functional purpose by controlling the drug release kinetics, thereby determining the therapeutic efficacy of the dosage forms. Producing these products with a high level of uniformity on a production scale, however, is challenging due to the inherent process complexities such as thermodynamics drying, spray atomisation and tablet mixing [1]. To better understand tablet mixing, early experimental studies have used a variety of techniques such as intermittent off-line weight gain analysis [2], colorimetry [3] and temperature difference [4]. Sensing using the terahertz radiation is fast gaining popularity [5-9]. In particular, in-line terahertz sensing has shown tremendous potential for pharmaceutical coating investigations [10-12]. In addition to being able to resolve the coating thicknesses of the pharmaceutical tablets at in-line setting, here we demonstrate how it can also be potentially used to study tablet mixing process by monitoring the sign of the acquired terahertz reflections.

\section{METHODOLOGY}

A 1.2 litre laboratory-sized perforated coating pan has been realised as shown in Figure 1 (a) and is used to perform tablet mixing trials. To closely mimic a commercial tablet mixing process, the coating pan is additionally fitted with tubular (trip bars) or ploughshare baffles, where the baffle design influences tablet mixing. In a manner similar to that described in [10], the coating pan is coupled to the TPI Imaga 2000 (TeraView Ltd, Cambridge, UK). Mixing trials of coated tablets are performed with bi-convex shaped tablets (caffeine cores) coated with Kollicoat IR Carmine and Kollicoat Smartseal (BASF, Ludwigshafen, Germany). The reason for the choice of the coating materials is that these coating materials have different refractive indices and colour thus resulting in different signs of terahertz reflection from the coating core interface. This in turn provides the possibility of distinguishing two kinds of tablet and evaluating the mixing efficiency for the TPI. The deconvolved off-line terahertz waveforms of these two types of tablets are shown in Figure 1 (b).

Based on a previously published analysis algorithm [13], we further develop it to classify two types of tablets by detecting the signs of the reflection resulting from the coatingcore interface, where it is anticipated that under a homogenous mixing scenario, it would result in an approximately 50-50 positive and negative detection ratio.
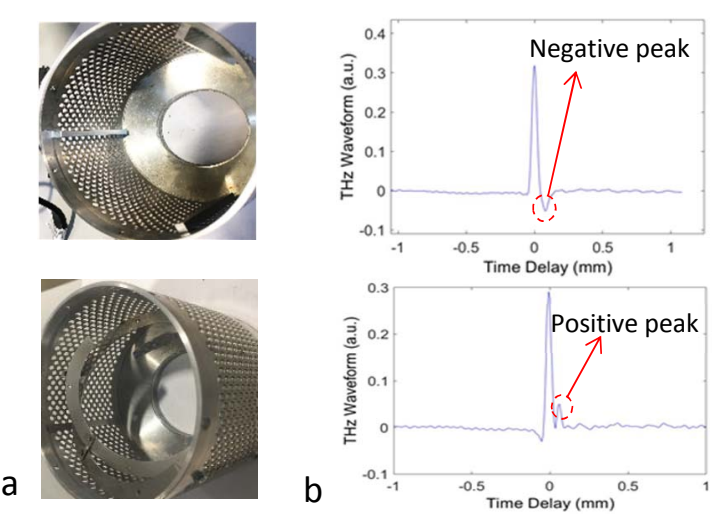

Fig. 1. (a) A photo of the laboratory scale pan coating pan fitted with tubular baffles (top) and ploughshare baffle (bottom). (b) Terahertz waveforms of two types of tablets with negative (top) and positive peaks (bottom) .

To test the data analysis algorithm for peak detection, we conducted three simple mixing trials: 1) an empty rotating coating pan without any tablets loaded; 2 ) only Carmine loaded and 3) only Smartseal tablets loaded. The entire process was measured using an in-line terahertz sensor with the TPI operating at $30 \mathrm{~Hz}$ acquisition rate. In these mixing trials, the coating pan was fitted with tubular baffles spaced out at $120^{\circ}$ apart. In order to compare against earlier work [14, 15], approximately 230 coated bi-convex shaped Kollicoat IR Carmine and Smartseal tablets were mixed and measured directly inside the coating pan rotating at $15 \mathrm{rpm}$. Measurement time was limited to a total of 10 minutes in order to reduce the amount of attrition to the dosage forms. We compare our experimental results with simulation results obtained numerically using the discrete element method (DEM) combined with a ray-tracing technique [15] due to a close similarity in the experiment conditions.

\section{RESULTS}

Using the modified analysis algorithm, this resulted in a detection rate as summarised in Table 1 . The results of the Smartseal trial are not included here as offline terahertz waveforms revealed that coatings applied in this particular batch were too thin to be accurately resolved by the TPI. Figure 2 shows two examples of deconvolved off-line terahertz waveforms where, unlike Fig. 1(b), no positive coating-core reflection can be observed. 

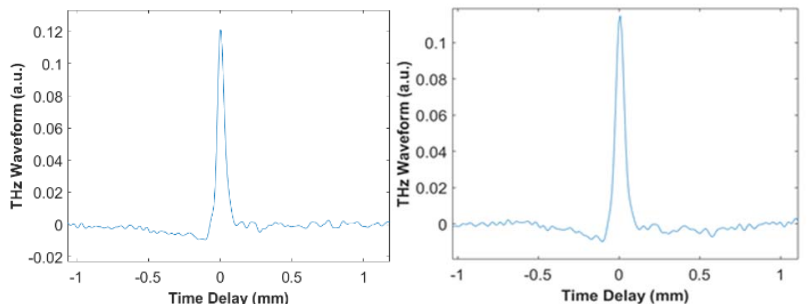

Fig. 2. Two offline terahertz waveforms from the Smartseal tablets batch used for inline mixing

For the other measurements, however, results are in agreement as what is expected. For example, in the absence of coated tablets, the total number of hits is 13 , significantly less than the Carmine trial and DEM simulation (see Figure 3). The reason why this number is not zero is due to the uncertainty involved in setting the magnitude of the thresholds for the peak detection of the coating reflection. For the same reason the detection accuracy is $\approx 90 \%$ for the measurement with only Carmine tablets. This is a fundamental limitation of the measurement and analysis algorithm. The total hit rates for the Carmine trial and simulation for the majority of the measurement time is approximately the same as what DEM predicted [15]. It is interesting to note that at the beginning of the mixing process the hit rate is $14 \%$ greater than the DEM prediction. This is possibly due to the use of smaller tablets in the experiments which results in slightly different different mixing dynamics. The resulting coating thickness distribution of the Carmine trial is shown in Figure 4 using an estimated refractive index of 1.86 from offline tablet TPI measurements (Figure 1 (b)). A description of the refractive index determination can be found in [12]. The resultant inter and intra-coating thickness distribution centers around 40-50 $\mu \mathrm{m}$.

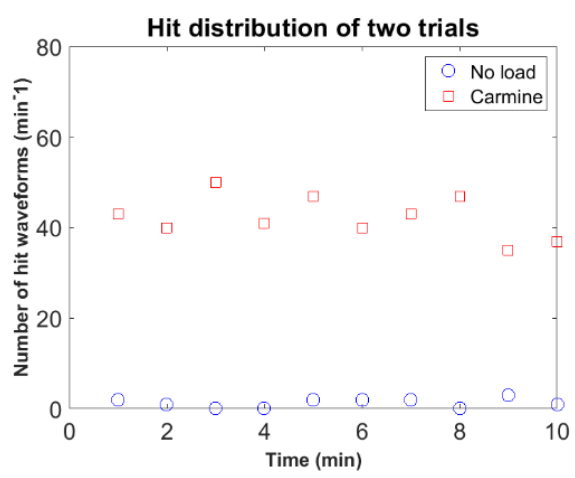

Fig. 3. Comparison of hit rates for no-load and Carmine tablets trials

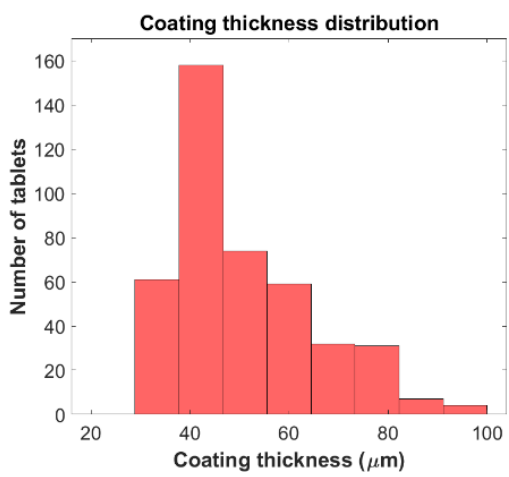

Fig. 4. Coating thickness distribution of in-line terahertz measurements for Carmine trial.

\section{SUMMARY}

We have successfully improved a previously published analysis algorithm to classify tablets on the basis of the strength of the coating-core reflections in order to detect the coating type. However due to the fact that Smartseal tablets in this particular batch did not have any resolvable positive peaks, we did not perform mixing trials. Future work aims to overcome this and apply the modified algorithm to study tablet mixing dynamics.

\section{ACKNOWLEDGEMENTS}

The authors acknowledge the financial support from UK Engineering and Physical Sciences Research Council Research Grant EP/R019460/1. The authors would also like to thank BASF and Colorcon for materials and helpful discussions.

\section{REFERENCES}

1. W. Ketterhagen et al., "Modeling tablet film-coating processes," in Predictive Modeling of Pharmaceutical Unit Operations: Elsevier, 2017, pp. 273-316.

2. G. L. Fourman, C. W. Hines, and R. S. Hritsko, "Assessing the uniformity of aqueous film coatings applied to compressed tablets," Pharmaceutical Technology, vol. 19, no. 3, pp. 70-76, 1995.

3. G. W. Smith, G. S. Macleod, and J. T. Fell, "Mixing efficiency in side-vented coating equipment," AAPS PharmSciTech, vol. 4, no. 3, pp. 71-75, 2003.

4. S. Tobiska and P. Kleinebudde, "A simple method for evaluating the mixing efficiency of a new type of pan coater," International Journal of Pharmaceutics, vol. 224, no. 1-2, pp. 141-149, 2001.

5. A.-K. Vynckier et al., "Calendering as a direct shaping tool for the continuous production of fixed-dose combination products via co-extrusion," European Journal of Pharmaceutics and Biopharmaceutics, vol. 96, pp. 125-131, 2015.

6. H. Lin et al., "Review of THz near-field methods," in Smart Structures, Devices, and Systems III, 2007, vol. 6414: International Society for Optics and Photonics, p. 64140L

7. D. YS Chau et al., "Determination of water content in dehydrated mammalian cells using terahertz pulsed imaging: a feasibility study," Current pharmaceutical biotechnology, vol. 17, no. 2, pp. 200-207, 2016.

8. H. Lin et al., "Through-substrate terahertz time-domain reflection spectroscopy for environmental graphene conductivity mapping," Applied Physics Letters, vol. 116, no. 2, p. 021105, 2020.

9. H. Lin et al, "Gas recognition with terahertz time-domain spectroscopy and spectral catalog: a preliminary study," in Terahertz Photonics, 2008, vol. 6840: International Society for Optics and Photonics, p. 68400X.

10. R. K. May et al., "Terahertz in-line sensor for direct coating thickness measurement of individual tablets during film coating in real-time," Journal of Pharmaceutical Sciences, vol. 100, no. 4, pp. 1535-1544, 2011.

11. H. Lin et al., "Measurement of the intertablet coating uniformity of a pharmaceutical pan coating process with combined terahertz and optical coherence tomography in-line sensing," Journal of Pharmaceutical Sciences, vol. 106, no. 4, pp. 1075-1084, 2017.

12. D. Alves-Lima et al., "Review of Terahertz Pulsed Imaging for Pharmaceutical Film Coating Analysis," Sensors, vol. 20, no. 5, p. 1441, 2020. 13. H. Lin et al., "Impact of processing conditions on inter-tablet coating thickness variations measured by terahertz in-line sensing," Journal of Pharmaceutical Sciences, vol. 104, no. 8, pp. 2513-2522, 2015.

14. H. Lin et al., "Steps towards numerical verification of the terahertz in-line measurement of tablet mixing by means of discrete element modelling," IET Microwaves, Antennas \& Propagation, vol. 12, no. 11, pp. 1775-1779, 2018.

15. C. Pei et al., "A quantitative comparison of in-line coating thickness distributions obtained from a pharmaceutical tablet mixing process using discrete element method and terahertz pulsed imaging," Chemical Engineering Science, vol. 192, pp. 34-45, 2018. 